\title{
Membrane Stabilizing Action of NCO-650 and Its Congeners
}

\author{
Kasumi ARAKAWA, Mayumi TONOOKA, Hiroshi GOTO \\ and Koji SAKAMOTO* \\ Department of Anesthesiology, University of Kansas Medical Center. \\ Kansas City, Kansas 66103, U.S.A. \\ * Department of Pharmacology. Showa University School of Medicine, \\ Sinagawa-ku, Tokyo 142. Japan \\ Accepted July 13, 1984
}

\begin{abstract}
NCO-650 and its congeners and two other antiallergics, disodium cromoglycate and tranilast, were studied to determine the degree of protection of rat erythrocytes against hypotonic hemolysis, the reduction of the surface tension of dipalmitoylphosphatidylcholine (DPPC) monolayer and the depression of the phase-transition temperature of DPPC liposome bilayers. NCO-650 was found to show the greatest hemolysis protection, reduction of the surface tension and depression of the phase-transition temperature, indicating that it possesses a significant affinity to cell membranes and a significant ability to stabilize cell membranes. Disodium cromoglycate and tranilast showed neither cell membrane affinity nor hemolysis protection, although they inhibit histamine release from mast cells like NCO-650 and its congeners. The significant membrane stabilizing action of NCO-650 must be related, at least in part, to its extraordinarily high lipid solubility.
\end{abstract}

It has generally been accepted that antiallergics exert their functions by inhibiting the release of chemical mediators and/or suppressing the overreactions of affected cells. Antihistamines, the most popular antiallergics, are known to work by competing with histamine at the sites of $\mathrm{H}_{1}$ receptors of affected cells. On the other hand, disodium cromoglycate (DSCG) and tranilast $\left(\mathrm{N}-5^{\prime}\right)$ exert their antiallergic actions by inhibiting the release of histamine and other mediators such as slow reacting substance- $A$ from mast cells $(1,2)$. Similarly, new drugs such as NCO-650 (p-tert-butyl-phenyl trans-4guanidinomethylcyclohexane carboxylate hydrochloride) and its congeners have been found to possess the ability to inhibit histamine release from isolated rat mast cells (3). We were interested in investigating NCO-650 and its congeners and other antiallergics (DSCG and $\mathrm{N}-5^{\prime}$ ) in terms of cell membrane stabilizing action and cell membrane affinity, by utilizing rat erythrocyte membranes and model cell membranes of phospholipid monolayers and bilayers.

\section{Materials and Methods}

1. Chemicals: Figure 1 shows the chemical structure and generic names of the drugs used in this study. As shown, NCO-650 and its congeners are tranexamic acid derivatives. They were generously supplied by Nippon Chemiphar.

2. Hypotonic hemolysis study: Protective and lytic effects of drugs on hypotonic hemolysis were performed by a modified Seeman's method (4). Male, 6-week old Sprague-Dawley rats weighing 160-170 g were anesthetized with pentobarbital (40 $\mathrm{mg} / \mathrm{kg}$, i.p.), the abdomen was opened, and blood was collected from the inferior vena cava with a heparinized syringe. The blood was centrifuged ( $2500 \mathrm{rpm} \times 15 \mathrm{~min}$ ) and the erythrocyte-rich precipitate was washed three times with isotonic phosphate-buffered saline (PBC: $142 \mathrm{mM} \mathrm{NaCl}$ and $10 \mathrm{mM}$ phosphate, $\mathrm{pH}$ 7.4). The concentration of erythrocytes in PBC was then adjusted to $4 \times 10^{9}$ cells $/ \mathrm{ml}$, using a Thoma's counting chamber, and this erythrocyte suspension was kept at $0^{\circ} \mathrm{C}$ 

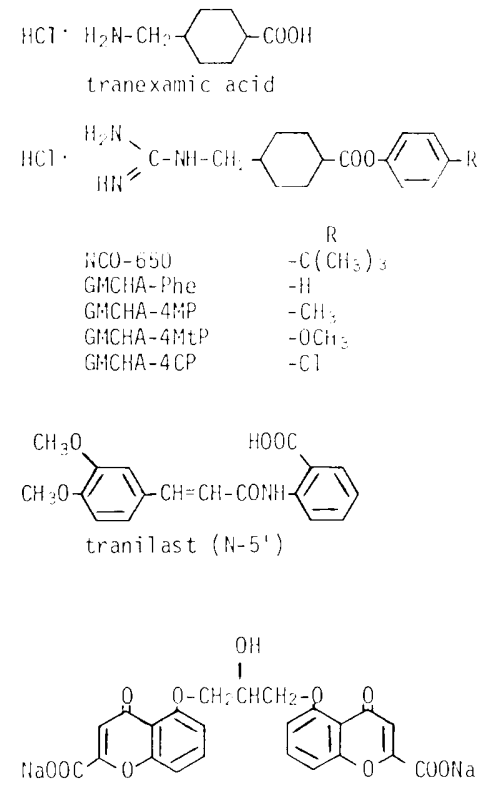

disodium cromoglycate (DSCG)

Fig. 1. Chemical structures and generic names of drugs studied. Abbreviations stand for the following: NCO-650: p-tert-butyl-phenyl trans-4-guanidinomethylcyclohexane carboxylate hydrochloride. GMCHA: trans-4-guanidinomethylcyclohexane carboxylate hydrochloride, GMCHA-4MP: 4-methylphenyl, GMCHA-4CP: 4-chlorophenyl, GMCHAPhe: phenyl, GMCHA-4MtP: 4-methoxyphenyl.

before use. The hypotonic buffer solution which would cause hemolysis of $70 \%$ of the erythrocytes was prepared by adjusting the concentration of sodium chloride in $10 \mathrm{mM}$ phosphate buffer ( $\mathrm{pH}$ 7.4) for each erythrocyte suspension. Since most of the drugs studied were water-insoluble, each drug was first dissolved in dimethylsulfoxide (DMSO) and was introduced into the hypotonic buffer solution to produce concentrations from 1 to $10,000 \% \mathrm{M}$. The final concentration of DMSO was always less than $1 \%$ volume, and this had been previously found not to affect the degree of hemolysis. Aliquots of $4.8 \mathrm{ml}$ of the hypotonic buffer solution in which various concentrations of each drug were dissolved and aliquots of $0.2 \mathrm{ml}$ of the erythrocyte suspension were pre-incubated $\left(37^{\circ} \mathrm{C}, 5\right.$ $\min )$. They were then mixed in glass test tubes and incubated for one hour in a $37^{\circ} \mathrm{C}$ water bath. Following incubation, this suspension was centrifuged (2000 rpm $\times 5$ $\min )$, and the free hemoglobin in the supernatant was determined at a wavelength of $540 \mathrm{~nm}$ using a Shimadzu spectrophotometer. The relative degree of hemolysis was expressed as a percentage of the control value of 70 percent (the hypotonic solution without a drug would produce $70 \%$ hemolysis) and plotted against the logarithmic concentrations of each drug. In addition, the hypotonic hemolysis study was performed for NCO-650 at different $\mathrm{pH}$ values $(5.7$ and 8.0 , in addition to $\mathrm{pH} 7.4$ ).

3. Surface tension study: Each drug was first dissolved in DMSO and then added to $100 \mathrm{mM}$ phosphate buffer solution to produce concentrations of 0.01 to $100 / \mathrm{M}$. This buffer solution was introduced into the trough of an Acoma Wilhelmy surface balance. Dipalmitoylphosphatidylcholine (DPPC) dissolved in a solvent consisting of absolute ethanol and purified petroleum $(1: 9 \mathrm{~V} / \mathrm{V})$ was spread on the surface of the buffer solution with a microsyringe to form one molecule in $100 \AA^{2}$. Surface tension was measured by the platinum hanging plate method (5) and recorded with a Riken Denki F-3EP X-Y recorder. The final concentration of DMSO was always less than $1 \%$ volume, which was found to have a negligible effect on the surface tension. The surface tension (dyne/ $\mathrm{cm}$ ) was plotted with respect to the concentrations of each drug on a logarithmic scale. In addition, surface tension was measured for NCO-650 when the $\mathrm{pH}$ of the buffer solution was at 5.7 and 8.0.

4. Phase-transition temperature study: DPPC was dispersed in $10 \mathrm{mM}$ of phosphate buffer ( $\mathrm{pH}$ 7.40) at a concentration of $1 \mathrm{mM}$ using a Bronson sonifier cell destructor 200 at a temperature above the phase transition of DPPC, for $20 \mathrm{~min}$. This method has been generally accepted to produce single bilayer liposomes of DPPC. NCO-650 and its congeners were then dissolved in the DPPC dispersion at various concentrations up to $5 \mathrm{mM}$. Aliquots of $3 \mathrm{ml}$ of this solution were introduced into a $10 \mathrm{~mm}$ light-path fluorometry cuvette, which was then placed in the thermostatted double-beam cuvette compartment of a Hitachi fluorophotometer. The temperature inside the cuvette was measured 
to the accuracy of $0.01{ }^{\circ} \mathrm{C}$ with a Takara thermister probe inserted into the cuvette and recorded with a Hitachi $057 X-Y$ recorder. The temperature was increased at a rate of $0.5^{\circ} \mathrm{C} / \mathrm{min}$ by circulating water from a Shimadzu water thermobath. Turbidity was measured by 90 degree light scattering at a wavelength of $360 \mathrm{~nm}$ and recorded by a strip chart recorder. The midpoint between the beginning of the decrease in the scattered light and the point where the scattering reached its plateau was taken as the phasetransition temperature for each concentration of NCO-650 and its congeners. A decrease in phase transition temperature from the control temperature without drugs was plotted against the concentration of each drug. From the slope of this graph, the concentration of each drug necessary to induce $1{ }^{\circ} \mathrm{C}$ of phase-transition depression was calculated. By applying the Van't Hoff equation (6), the DPPC liposome/water partition coefficient was estimated to compare the lipid affinity.

\section{Results}

1. Hypotonic hemolysis study: NCO-650 and its congeners showed biphasic effects on hypotonic hemolysis: at lower concentrations, they protected erythrocytes, and at higher concentrations, they exerted a lytic effect on the erythrocytes. (Fig. 2). Tranexamic acid, from which NCO-650 and its congeners were derived, and disodium cromoglycate (DSCG) did not show any effect on hypotonic hemolysis. On the other hand, tranilast $\left(\mathrm{N}-5^{\prime}\right)$ showed only a lytic effect on the erythrocytes at concentrations higher than about $60 \mu \mathrm{M}$. The concentrations which exerted the maximum protection for erythrocytes against $70 \%$ hypotonic hemolysis were about $40 \mu \mathrm{M}, 200 \mu \mathrm{M}$ and $500 \mu \mathrm{M}$ for NCO-650, GMCHA-4CP and GMCHA-4MP. respectively, and near $2000 \mu \mathrm{M}$ for $\mathrm{GMCHA}$ Phe and GMCHA-4MtP.

Increasing the $\mathrm{pH}$ of the buffer solution decreased the concentration of NCO-650 required to produce the maximal protective effect against hypotonic hemolysis (Fig. 3). The concentrations were about 100, 40 and 30 , $\mathrm{M}$ when the $\mathrm{pH}$ of the buffer solution was 5.7, 7.4 and 8.0, respectively.

2. Surface tension study: NCO-650 and its congeners decreased the surface tension of the DPPC monolayer in such a way as to produce reverse sigmoid curves (Fig. 4). At the concentration of $100 \mu \mathrm{M}$, the surface tensions were 20,30,36, 40 and 41 dyne/cm for NCO-650, GMCHA-4CP, GMCHA-4MP, GMCHA-4MtP and GMCHA-Phe, respectively. On the other hand, DSCG and N-5' did not decrease the surface tension

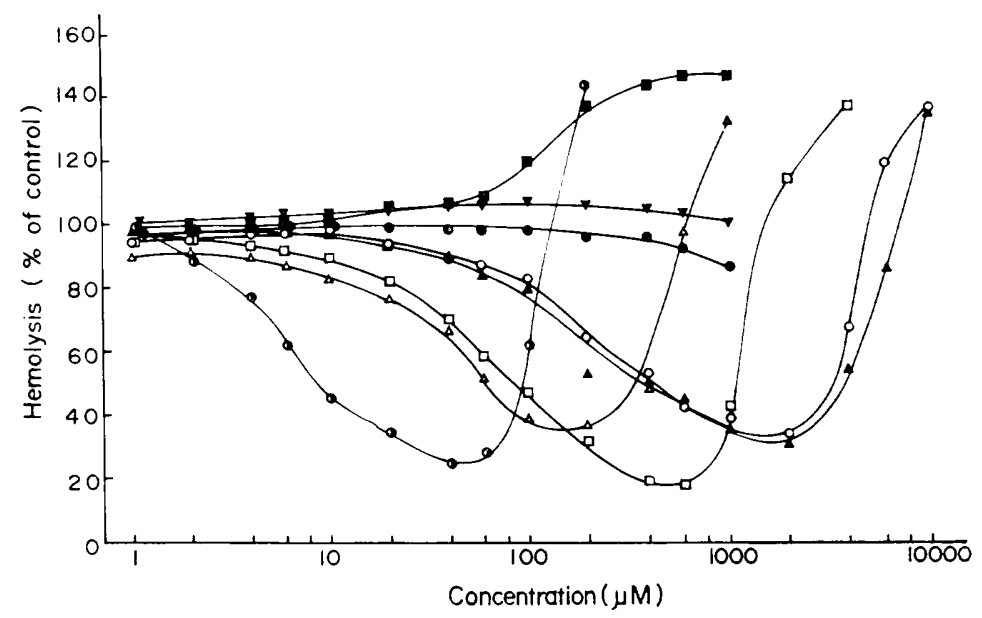

Fig. 2. Effect of drug on $70 \%$ hypotoric hemolysis of rat erythrocytes. Relative degree of hemolysis is expressed as a percentage of the control value and shown on the ordinate. $\triangle \mathrm{NCO}-650, \triangle \longrightarrow \triangle \mathrm{GMCHA}-4 \mathrm{CP}, \square-\square \mathrm{GMCHA}-4 \mathrm{DP}, \mathrm{O} \longrightarrow \mathrm{GMCHA}-\mathrm{Phe}$, 


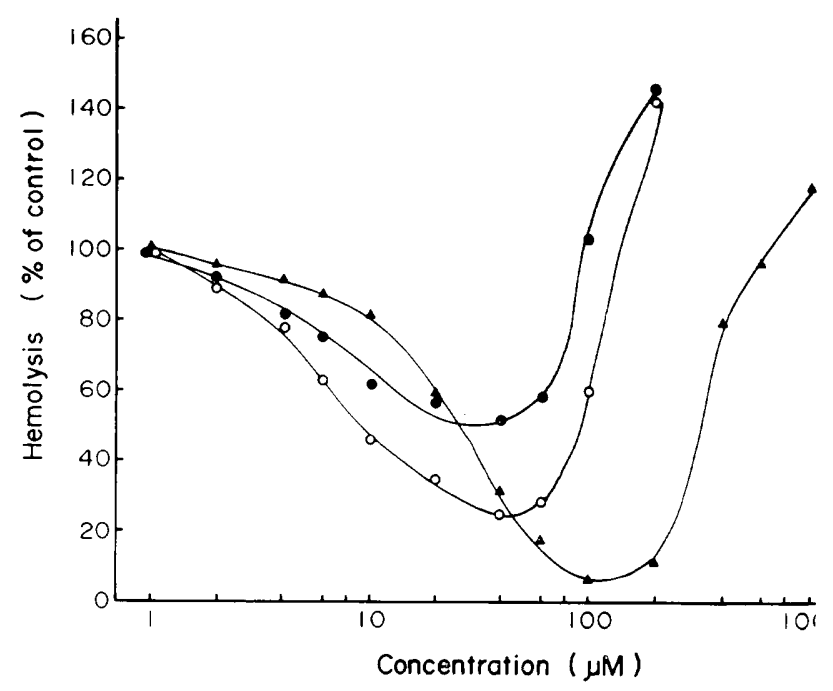

Fig. 3. Effect of pH on $70 \%$ hypotonic hemolysis by NCO-650.

$\Delta-\mathbf{p H} 5.7 .0-\mathrm{OpH} 7.4$. - $\mathrm{pH} 8.0$.

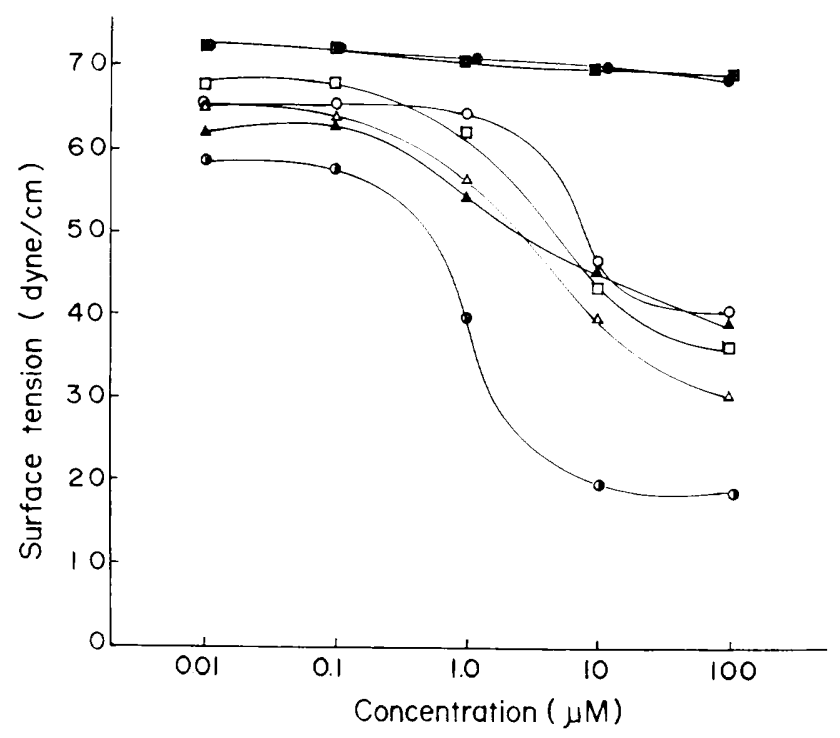

Fig. 4. Effect of drug on surface tension of DPPC monolayer. - NCO-650, $\triangle \triangle \mathrm{GMCHA-}$ 4CP. $\square \square$ GMCHA-4MP, $O-O$ GMCHA-Phe, $\triangle \mathrm{GMCHA}-4 \mathrm{M}+\mathrm{P}$. $N-5^{\prime}$

measurably. Increasing the $\mathrm{pH}$ of the buffer solution caused a $\mathrm{pH}$-dependent decrease in surface tension produced by NCO-650 (Fig. 5).

3. Phase-transition temperature study: NCO-650 showed the steepest slope in the relationship between the depression of phase transition temperature and concentration (Fig. 6). The slopes for GMCHA-4CP and GMCHA-4MP were next in steepness, and those for GMCHA-Phe and GMCHA-4MtP were the least steep. The calculated concentration of NCO-650 necessary to induce $1^{\circ} \mathrm{C}$ depression of the phase-transition temper- 


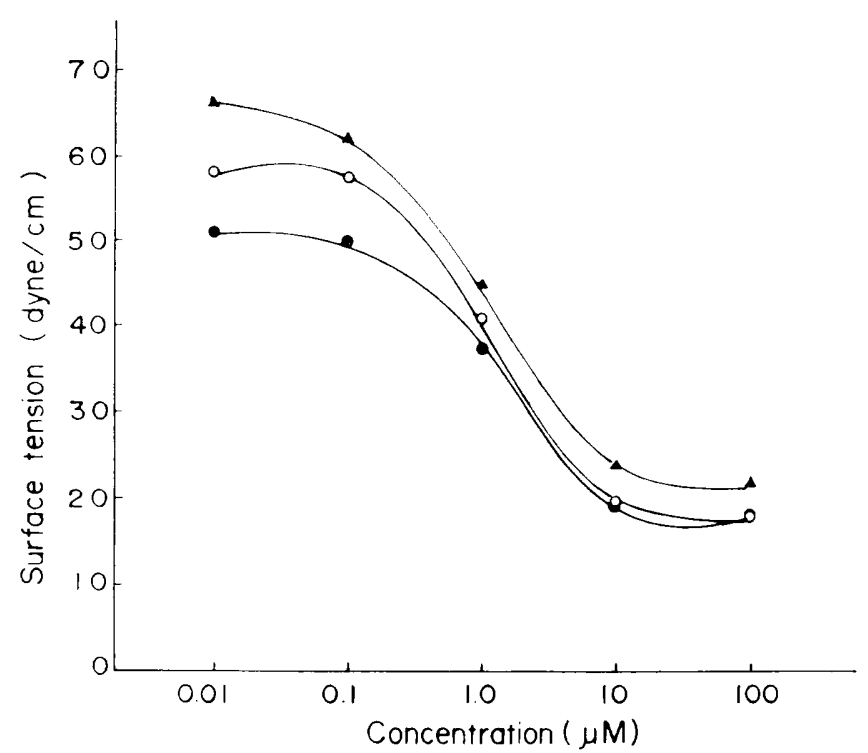

Fig. 5. Effect of $\mathrm{pH}$ on surface tension by NCO-650. pH 8.0 .

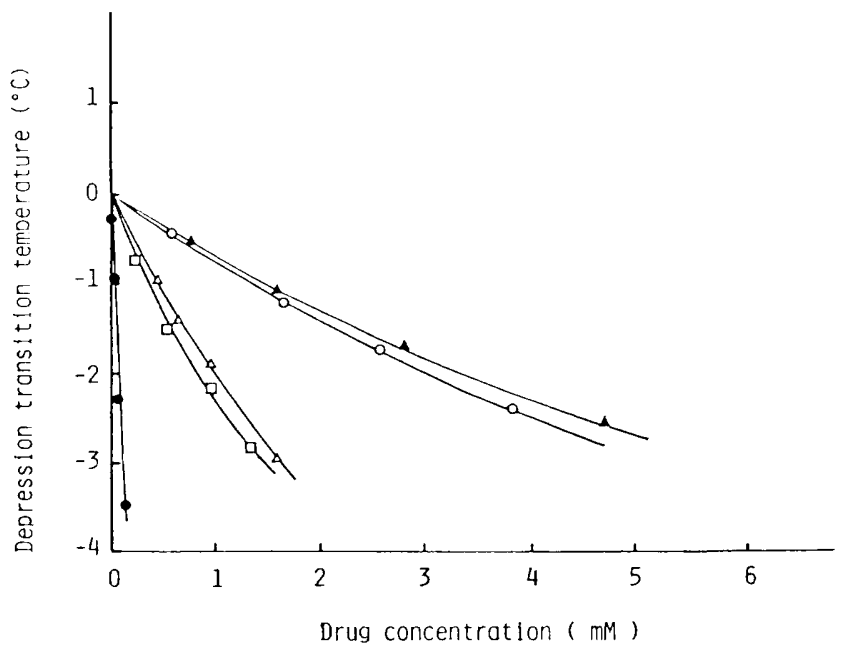

Fig. 6. Effect of NCO-650 and its congeners on phase transition temperature of $1 \mathrm{mM}$ DPPC liposome suspension. The ordinate indicates a decrease in temperature form controlled phase-transition temperature. $\mathrm{NCO}-650, \triangle \longrightarrow \triangle \mathrm{GMCHA}-4 \mathrm{CP},-\square \mathrm{GMCHA}-4 \mathrm{MP}, \mathrm{O}-\mathrm{O}$ GMCHA-Phe, $\Delta-\mathbf{\Delta}$ GMCHA-4MtP.

ature was only $3.81 \times 10^{-2} \mathrm{mM}$, which was one order smaller than the next value, $4.06 \times 10^{-1} \mathrm{mM}$ of GMCHA-4MP. (Table 1). The estimated partition coefficient (K) between the DPPC liposome membrane and water for $\mathrm{NCO}-650$ was $6.38 \times 10^{3}$, and this was 53.7-fold larger than the next large value, for GMCHA-4MP $\left(1.10 \times 10^{2}\right)$.

\section{Discussion}

Inhibition of the release of histamine from mast cells by some of the antiallergics has been shown to be related to their effects on cell membrane stabilization $(3,7)$. The 
Table 1. Concentration of NCO-650 and its congeners to induce $1^{\circ} \mathrm{C}$ depression of the phase-transition temperature in $1 \mathrm{mM}$ DPPC suspension and partition coefficients, $K$, between DPPC vesicles and water

\begin{tabular}{ccc}
\hline Drug & Concentration $(\mathrm{mM})$ & $\mathrm{K}$ \\
NCO-650 & $3.81 \times 10^{-2}$ & $6.38 \times 10^{3}$ \\
GMCHA-4MP & $4.06 \times 10^{-1}$ & $1.10 \times 10^{2}$ \\
GMCHA-4CP & $5.10 \times 10^{-1}$ & $9.29 \times 10$ \\
GMCHA-Phe & 1.49 & $3.05 \times 10$ \\
GMCHA-4MtP & 1.59 & $2.85 \times 10$ \\
\hline
\end{tabular}

protective effects of NCO-650 and its congeners against hypotonic hemolysis of rat erythrocytes indicate the ability of these compounds to stabilize eyrthrocyte membranes. The basic structure of membranes of cells, including erythrocytes, consists of phospholipid bilayers with a hydrophilic portion outside and a lipophilic portion inside (8). NCO-650 and its congeners possess a lipophilic phenyl ester group which must facilitate penetration of the hydrophilic portion of the phospho!ipid bilayer and to partition in the cell membranes. In addition to the phenyl ester, NCO-650 possesses a lipophilic tertiary butylphenyl group which must enhance its penetration into the erythrocyte membranes and increase its concentration in the membrane phase. This is probably why NCO-650 exerted the maximal hemolysis protection at the lowest concentration. This is similar to the fact that the more lipid soluble volatile anesthetics (as well as local anesthetics) are more effective in stabilizing the nerve cells $(9,10)$. Moreover, all lipid soluble anesthetics protect erythrocytes from hypotonic hemolysis, and the degree of anti-hemolysis correlates well with the degree of nerve blocking (11). DSCG and $N-5^{\prime}$ did not show the protective effect on hypotonic hemolysis, indicating that their lipid solubilities are extremely low. Thus, their inhibition of histamine release from mast cells may not be simply related to the cell membrane stabilization; instead, it may be related to the prevention of calcium ion influx (12). The pKa of NCO-650 is said to be higher than 8.0. Thus, most of the molecules are in a protonated, charged form at $\mathrm{pH} 5.7$, where NCO-650 exerted its maximum hemolysis protection $(95 \%)$, at the bulk concentration of about $100 \mu \mathrm{M}$. On the other hand, at $\mathrm{pH}$ 8.0. NCO-650, with an increased proportion of uncharged, base form, protected erythrocytes only $50 \%$ maximally, but at much lower bulk concentration (30 $\mu \mathrm{M})$. These observations suggest that uncharged as well as charged forms of NCO-650 are absorbed in the cell membranes, causing protective (at lower concentrations) as well as lytic effects (at higher concentrations) on the erythrocytes. It is also suggested that uncharged forms of NCO-650 are more lipophilic, thus possess a higher affinity for cell membranes, and causing the lytic action on erythrocytes at much lower bulk concentrations. DPPC is a major component of cell membranes, and its monolayers have been used for many years as models to investigate the interactions between drugs and cell membranes (13). A decrease in surface tension shown by NCO650 and its congeners indicates that molecules of these compounds penetrate and partition in an interface between air and surface monolayers. NCO-650 decreased the surface tension more than any of its congeners correlating with its high lipid solubility and its high affinity for the membrane. This phenomenon is similar to that shown by potent local anesthetics, which are more lipophilic, and decrease surface tension more (14). DSCG and N-5' did not exert significant surface activities indicating again that their ability to inhibit histamine release from mast cells is probably not related to membrane stabilization.

The surface tension curve has a reverse sigmoid shape and reaches plateau as concentrations increase. It is speculated that empty space available at the surface unoccupied by the DPPC monolayer has been saturated maximally, and further increase in bulk concentration does not decrease the surface tension further. Increasing the $\mathrm{pH}$ of the buffer facilitated the action of NCO-650 
in decreasing surface tension. This again suggests that the uncharged form of $\mathrm{NCO}$ 650 has a higher affinity to the membranes. owing to its higher lipophilicity.

The model membranes of DPPC liposomes are in crystalline phase below and in liquid crystalline phase above the phase-transition temperature $\left(41^{\circ} \mathrm{C}\right)$. They undergo transition between two phases according to temperature, which is affected by the chemical environment. Depression of the phasetransition temperature of DPPC liposomes by NCO-650 and its congeners indicates that molecules of these agents penetrate and partition in the bilayers and are fluidizing the bilayer membranes. An increase in fluidity of phospholipid bilayers indicates the expansion of the bilayer membranes $(4,15)$. The membrane expansion by NCO-650 and its congeners may be the reason for the stabilization of the erythrocyte membrane, by increasing the critical hemolytic volume, and possibly by decreasing the membrane permeability. The volume of erythrocytes can increase because of cell membrane expansion. Therefore, more water is allowed to remain in the erythrocytes before the lysis will occur. Besides, it can be speculated that the increase in membrane fluidity (membrane expansion) to some extent might decrease the permeability for some substances such as hemoglobin since both hydrophilic and lipophilic portions of phospholipid bilayers increase their mobility and also become less aligned. However, it is important to realize that if the fluidity of the cell membrane is overly increased, cell membranes become overexpanded and become unstable as indicated in the hypotonic hemolysis study in which NCO-650 and its congeners exerted a lytic effect on the erythrocytes at higher concentrations. The fact that a significantly low concentration is required to suppress the phase-transition temperature indicates that NCO-650 has a marked stabilization effect on the cell membranes as compared with its congeners. This is again similar to the action of potent local anesthetics such as tetracaine. which markedly depress the phase-transition temperature of DPPC bilayers and increase the stabilization of the cell membrane, as opposed to weak local anesthetics such as procaine, where membrane expansion is not as great (16).

The calculated partition coefficients between DPPC liposome and water are in the order NCO-650》, GMCHA-4MP and GMCHA-4CP >, GMCHA-Phe and GMCHA$4 \mathrm{MtP}$, and correlate well with both the degree of protection for erythrocytes against hypotonic hemolysis and with the degree of the reduction of surface tension. Therefore, the potent action of NCO-650 on cell membranes must indeed be due at least in part to its extraordinarily high membrane affinity.

In summary, the newly developed tranexamic acid derivative NCO-650 possesses an ability to produce cell membrane stabilization at very low concentrations. Although the strong effect of NCO-650 on the cell membrane stabilization may be related to its ability to inhibit histamine release, the usefulness of NCO-650 as an antiallergic agent remains to be investigated.

\section{References}

1 Cox, J.S.G.: Disodium cromoglycate (FPL 670) ('Intal'): A specific inhibitor of reaginic antibodyantigen mechanisms. Nature 216, 1328-1329 (1967)

2 Koda, A., Nagai, H., Nakayama, S., Inoue, K. and Nakamura, K.: Experimental asthma in rats, and the effect of $\mathrm{N}\left(3^{\prime} 4^{\prime}\right.$-dimethoxycinnamoyl) anthranilic acid $\left(\mathrm{N}-5^{\prime}\right)$. Folia Pharmacol. Japon. 74, 699-709 (1978) (Abs. in English)

3 Akagi, M., Mio, M. and Tasaka, K.: Histamine release inhibition and prevention of the decrease in membrane fluidity induced by certain antiallergic drugs: Analysis of the inhibitory mechanism of NCO-650. Agents Action 13, 149-156 (1983)

4 Seeman, P.: A method for distinguishing specific from nonspecific hemolysins. Biochem. Pharmacol. 15, 1767-1774 (1966)

5 Lin, H., Ueda, I., Lin, S.H., Shieh, D.D., Layama, $H$. and Eyring, $H$.: Surface activities of tertiary amine local anesthetics at air/water interface in the presence and absence of phospholipid monolayers. Biochim. Biophys. Acta 598, 51-65 (1980)

6 Kayama, H., Kaneshina, S. and Ueda, I.: Partition equilibrium of inhalation anesthetics and alcohols between water and membranes of phospholipids with varying acyl chain-lengths. Biochim. Biophys. Acta 646, 135-142 (1981) 
7 Ennis, M., Truneh, A., White, J.R. and Pearce, F.L.: Inhibition of histamine secretion from mast cells. Nature 289, 186-187 (1981)

8 Singer, S.J. and Nicolson, G.L.: The fluid mosaic model of the structure of cell membranes. Science 175, 720-731 (1972)

9 Covino, B.G. and Vassallo, H.G.: Local Anesthetics: Mechanisms of Action and Clinical Use. Grune and Stratton. New York (1976)

10 Tanifuji, Y., Eger, E.I., II and Terell, R.C.: Some characteristics of an exceptionally potent inhaled anesthetic: Thiomethoxyflurane. Anesth. Analg. (Cleve.) 56, 387-390 (1977)

11 Seeman, P.: The membrane actions of anesthetics and tranquilizers. Pharmacol. Rev. 24, 583-655 (1972)

12 Spataro, A.C. and Bosman, H.B.: Mechanism of action of disodium cromoglycate-mast cell calcium ion influx after a histamine-releasing stimulus. Biochem. Pharmacol. 25, 505-510 (1976)

13 Ueda, I., Shieh, D.D. and Eyring, H.: Anesthetic interaction with a model cell membrane: Expansion, phase transition, and melting of the lecithin monolayer. Anesthesiology 41, 217-225 (1974)

14 Vilallonga, F.A. and Phillips, E.W.: Surface activities of procaine, lidocaine, and tetracaine and their interaction energies with phospholipid monolayers. J. Pharm. Sci. 68, 314-316 (1979)

15 Isomaa, B.: Interactions of surface-active alkyltrimethyl-ammonium salts with the erythrocyte membrane. Biochem. Pharmacol. 28, 975-980 (1979)

16 Ueda, I.. Tashiro, C. and Arakawa, K.: Depression of phase-transition temperature in a mode! cell membrane by local anesthetics. Anesthesiology $46,327-332$ (1977) 